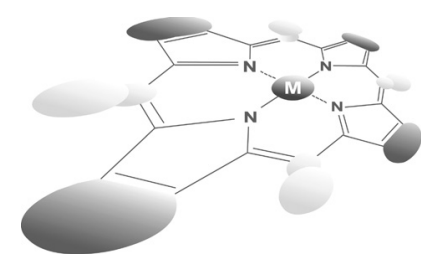

\title{
Tuning the morphology of mesoscopic structures of porphyrin macrocycles functionalized by an antimicrobial peptide
}

\author{
Rita Cimino ${ }^{a}$, Elisa Grellonia, Gabriele Magnaa, Donato Montia, \\ Manuela Stefanellia, Emanuela Gatto ${ }^{a}$, Ernesto Placidi ${ }^{b}$, \\ Francesca Biscaglia ${ }^{c}$, Marina Gobbo ${ }^{c}$ and Mariano Venanzi ${ }^{\star a}$
}

\author{
${ }^{a}$ Dept. of Chemical Science and Technologies, University of Rome Tor Vergata, via della Ricerca Scientifica 1, \\ 00133 Rome, Italy \\ ${ }^{\mathrm{b}}$ Dept. of Physics, University of Rome 'La Sapienza', P.le Aldo Moro 5, 00185 Rome, Italy \\ ${ }^{c}$ Dept. of Chemical Sciences, University of Padua, via F. Marzolo, 35131 Padua, Italy
}

Dedicated to Professor Roberto Paolesse on the occasion of his 60th birthday.

Received 15 November 2019
Accepted 16 December 2019

\begin{abstract}
The aggregation properties of two peptide-porphyrin conjugates were investigated by optical spectroscopy and microscopy imaging with nanometer resolution. Specifically, a tetraphenylporphyrin platform was functionalized by (L)-magainin, a 23-residue long antimicrobial peptide, and by a (L)magainin analogue differing from the parent peptide by a single residue substitution, i.e. an Ala vs. Phe replacement in the position 5 of the peptide chain. Spectroscopic and microscopy results show that this single-site substitution has a small effect on the secondary structure attained by the two peptide analogues, but deeply affects the morphology of the mesoscopic structures deposited on hydrophilic mica from methanol/water solutions. In particular, only the Ala-substituted peptide-porphyrin conjugate was shown to be able to form micrometric fibrils, coating homogeneously a hydrophilic mica surface. These results pave the way for potential applications of porphyrin-peptide compounds in localized photodynamic therapy and for designing solid-state stereoselective sensors.
\end{abstract}

KEYWORDS: chiral nanostructures, hierarchical self-assembly, hybrid materials, organic coating, porphyrin-peptide conjugates.

\section{INTRODUCTION}

Porphyrin-based materials have been deeply investigated as photosensitizing agents in photodynamic therapy (PDT) of tumors for their unique cytotoxic properties [1]. Recently, it has been proposed to use the same approach as an alternative strategy for antimicrobial therapy, due to the increasing resistance of bacterial infection to the available antibiotics [2]. PDT may secure a broad-band antimicrobial activity, exerting cytotoxic effects through nonspecific oxidative stress on multiple targets.

*Correspondence to: Mariano Venanzi, tel.: +39 0672594468 , fax: +390672594328, email: venanzi@uniroma2.it.
The charge of the photosensitizer (PS) is an important factor that determines the selective interaction of positively charged PS's against GRAM-negative bacteria. One strategy to enhance the activity of neutral PSs against GRAM-negative bacteria is to functionalize the PS agent with antimicrobial peptides (CAMPs). It has been shown that the cationic nature of CAMPs strongly promotes the interaction of hydrophobic PSs with prokariotic membranes [3-5].

Moreover, the affinity of cationic peptides for the membrane can be modulated by including hydrophobic residues in the peptide sequence, conferring amphiphilic character to the peptide. This property enhances the membrane permeabilization capacity of CAMPs and, therefore, their cytotoxic activity. 
Recently, some of us studied several covalently-linked peptide-porphyrin compounds for PDT applications $[6,7]$. Several CAMPs, i.e. apidaecin Ib, buforin II and magainin 2, were used to enhance the internalization of a porphyrin photosensitizer against target cells and to improve the solubility of hydrophobic porphyrins in a biological milieu. In particular, it was found that aggregation of TPP-magainin as large chiral supramolecular structures was promoted when the peptide attained a stable $\alpha$-helical conformation, suggesting a templating effect of the peptide secondary structure [8].

Aggregation of the peptide-porphyrin compounds was easily monitored by the marked bathochromic and hypsochromic shifts of the porphyrin absorption features and the sensible quenching of its fluorescence emission. A marked reduction of singlet oxygen production was also observed $[6,9]$.

Circular dichroism (CD) spectroscopic studies carried out in both the peptide and porphyrin absorption regions demonstrated that the configuration of the amino acid covalently bound to the macrocycle determined the chiral arrangement of the obtained supramolecular mesoscopic structure $[8,9]$. Interestingly, the CD spectrum of TPP(L)-magainin was characterized by an intense exciton splitting of the Soret transition band. The positive/ negative signature of the bisignate $\mathrm{CD}$ band suggested the anticlockwise winding of the excitonically coupled porphyrin units, organized in helical aggregates of mesoscopic size [10]. This finding emphasizes the role of the intrinsically chiral peptide chains to orient the winding of the helically arranged porphyrin macrocycles.

We have recently shown that the morphology of peptide aggregates in aqueous solutions are determined by the subtle balance of structural features, determining the conformational and dynamical properties of the peptide chain, and environmental (hydrophobic) effects [11].

In particular, we have observed that the formation of peptide fibrils is promoted by the population of helical conformation, and that this structural feature determines by hierarchical self-assembly the morphology of mesoscopic supramolecular architectures. A single amino acid substitution is sufficient to destroy the anisotropic 3D arrangement of the peptide aggregate, giving rise to the formation of globular structures as a result of predominant hydrophobic effects [12].

Recent studies carried out by our group have been focused on the effect of the presence of an amino acid residue and other chiral substituents on the periphery of related macrocycles. We have shown that such functionalization gave rise to the formation by hierarchical self-assembly of mesoscopic structures featuring supramolecular chirality $[13,14]$.

In this contribution, we report on the aggregation properties of two peptide-porphyrin compounds comprising a tetraphenylporphyrin unit suitably derivatized at the meso position with a carboxylic group for linking
(L)-magainin, a 23-residue antimicrobial peptide, or a magainin analogue characterized by a single Ala vs. Phe modification in the position 5 of the peptide chain. The two compounds, the molecular structures of which are reported in Chart 1, will be denoted in the following as TPPMag $(\mathrm{MW}=3470 \mathrm{~g} / \mathrm{mol})$ and TPPMagA5 $(\mathrm{MW}=$ $3547 \mathrm{~g} / \mathrm{mol}$ ), respectively.

The presence of charged and polar amino acids alternated to non-polar residues, together with the electron-rich, aromatic porphyrin platform conferred to the two compounds investigated a strong amphiphilic character that can be usefully exploited to study their aggregation properties in methanol/water solutions. It will be shown that a single-site substitution of an aromatic residue (Phe) with an apolar amino acid (Ala) deeply affects the 3D morphology of porphyrin-peptide aggregates of mesoscopic size.

\section{EXPERIMENTAL}

\section{Synthesis}

The synthesis of the peptide-porphyrin compounds here investigated has already been reported elsewhere [8]. Briefly, peptides were prepared on an automated Advanced Chemtech $348 \Omega$ peptide synthesizer, on a $0.05 \mathrm{mmol}$ scale, starting from preloaded Wang resins (resin substitution $0.51-0.72 \mathrm{mmol} \mathrm{g}^{-1}$ ). Fmoc deprotection was achieved with $20 \%$ piperidine in DMF. Couplings were performed in the presence of $O$-(benzotriazol1-yl) $N, N, N^{\prime}, N^{\prime}$-tetramethyluronium hexafluorophosphate/ $N$-hydroxybenzotriazole/ $N, N$-diisopropylethylamine or $O$-(7-azabenzotriazol-1-yl) $N, N, N^{\prime}, N^{\prime}$-tetramethyluronium hexafluoro-phosphate/ $N$-hydroxy-7-azabenzotriazole/ $\mathrm{N}, \mathrm{N}$-diisopropylethylamine (reaction time $45-60 \mathrm{~min}$ ), using an excess of 4 equivalents of the carboxyl component. For the conjugation to the porphyrin, the H-peptide-resin $(0.025 \mathrm{mmol})$ was swelled in DMF, the solvent was drained off and $600 \mu \mathrm{L}$ of a $\mathrm{DMF}-\mathrm{CH}_{2} \mathrm{Cl}_{2}$ solution $(1: 1 \mathrm{v} / \mathrm{v})$ containing $0.05 \mathrm{mmol}$ of TPP-OH, $0.05 \mathrm{mmol}$ diisopropylcarbodiimide and $0.05 \mathrm{mmol}$ 1-hydroxybenzotriazole was added.

\section{Spectroscopy and microscopy techniques}

Spectroscopic grade solvents $(\mathrm{MeOH}, \mathrm{DMSO}$ and $\mathrm{CHCl}_{3}$ ) were provided by Carlo Erba reagents (Milan, Italy), and used without further purification. Concentrations were determined by UV-vis absorption measurements at $\lambda=414 \mathrm{~nm}$ (Soret band), assuming a molar extinction coefficient $\varepsilon(414)=400000 \mathrm{M}^{-1} \cdot \mathrm{cm}^{-1}$ in methanol [14]. UV-vis absorption measurements were carried out on a CaryWin 100 spectrophotometer (Cary, USA), using quartz cells of appropriate path length (Hellma). Fluorescence measurements have been carried out with a Fluoromax-4 (Horiba) with single photon counting detection using quartz cells (Hellma). Circular 

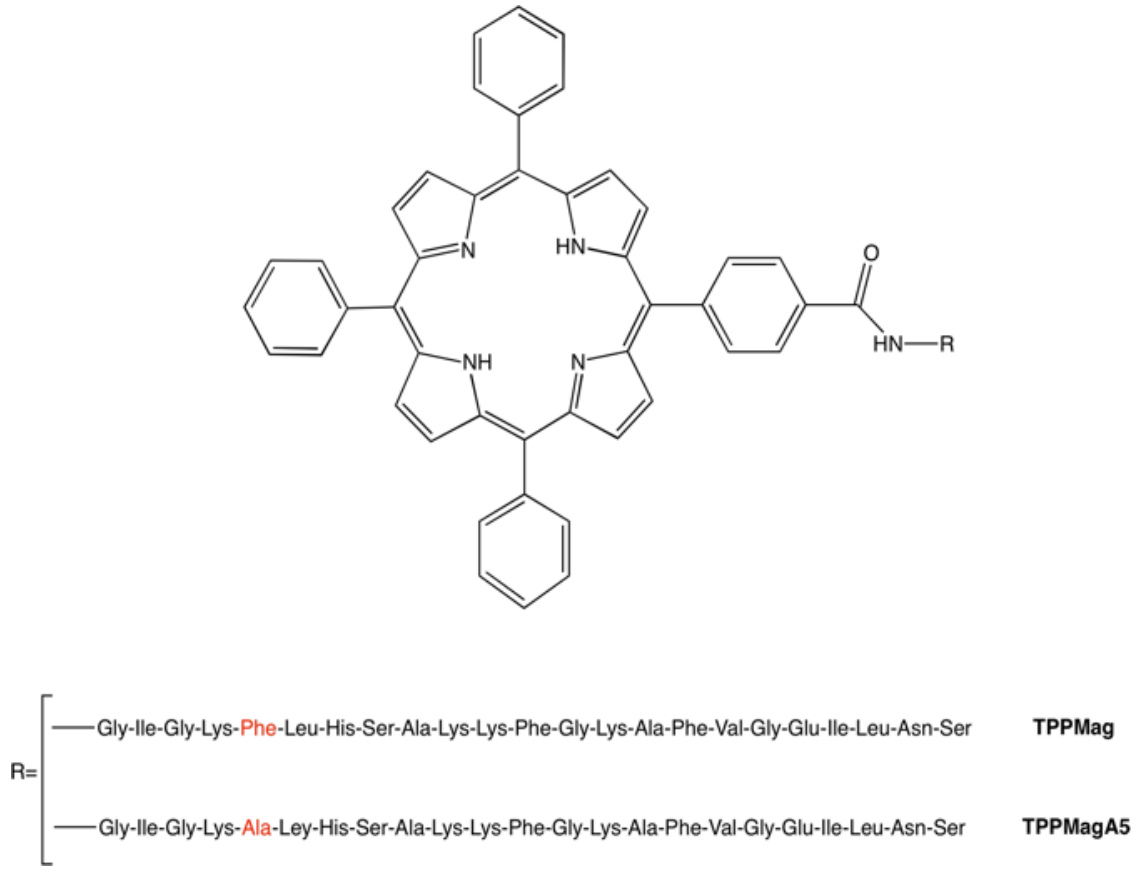

Chart 1. Molecular structures and acronyms of the investigated peptide-porphyrin conjugates.

Dichroism spectra were obtained by a JASCO J600 spectropolarimeter (JASCO, Japan). The reported spectra have been obtained by the average of 8 repeated spectra.

Atomic Force Microscopy experiments were carried out in air at room temperature on a Veeco Multiprobe IIIa instrument (Santa Barbara, CA). The AFM measurements were carried out in tapping mode on films obtained by drop casting micromolar methanol and methanol/water 30/70 (v/v) solutions of TPPMag and TPPMagA5 on mica and incubating for $18 \mathrm{~h}$ in a dryer. For the AFM measurements, a $\mathrm{Si}$ super sharp tip, functionalized by carbon flake was used (curvature $1 \mathrm{~nm}$, elastic constant $5 \mathrm{~N} / \mathrm{m}$, resonance frequency $150 \mathrm{kHz}$ ).

\section{RESULTS AND DISCUSSION}

\section{Spectroscopic characterization of peptide-porphyrin compounds in solution}

The spectroscopic properties of TPPMag and TPPMagA5 in methanol and methanol/water $(\mathrm{m} / \mathrm{w})$ mixtures were characterized by UV-vis absorption, steady-state fluorescence and circular dichroism (CD) experiments. In the former solvent the absorption spectra of the two compounds in the visible region are dominated by the porphyrin absorption bands, i.e. the intense Soret band at $\lambda_{\max }=414 \mathrm{~nm}\left[\mathrm{~S}_{0} \rightarrow \mathrm{S}_{2}, \varepsilon(414)=\right.$ $\left.4.0 \cdot 10^{5} \mathrm{~mol}^{-1} \mathrm{~cm}^{-1}\right]$, and the four $\mathrm{Q}$ bands $\left[\mathrm{S}_{0} \rightarrow \mathrm{S}_{1}\right.$, $\left.\varepsilon(515)=1.0 \cdot 10^{4} \mathrm{~mol}^{-1} \mathrm{~cm}^{-1}\right]$ at $\lambda_{\text {max }}=515,550,590$ and $645 \mathrm{~nm}$, typical of free-base porphyrins. In the case of TPPMag the Q-band absorptions appear to be slightly shifted to longer wavelengths, i.e. $\lambda_{\max }=513,549,588$ and $644 \mathrm{~nm}$. For both compounds the fluorescence emission spectra showed a single emission band peaked at $\lambda_{\max }=646 \mathrm{~nm}$.

The aggregation of TPPMag and TPPMagA5 at micromolar concentration was promoted by exploiting the hydrophobic effect exerted by increasing the fraction of water in $\mathrm{m} / \mathrm{w}$ solutions. Figure $1 \mathrm{~A}$ shows the UV-vis absorption spectra of TPPMagA5 $(6.6 \mu \mathrm{M})$ in methanol and in 70/30, 50/50 and 30/70 (v/v) m/w solutions. Upon increasing the content of water in the $\mathrm{m} / \mathrm{w}$ solutions, a significant decrease of the intensity and a remarkable broadening of the Soret absorption band were observed. The red shift of the absorption band at high water content strongly suggests the formation of J-type aggregates, i.e. ground-state complexes characterized by a head-to-tail stacking of the interacting porphyrin macrocycles [15].

The absorption spectrum of TPPMag in 30/70 (v/v) $\mathrm{m} / \mathrm{w}$ solution also revealed a similar intensity decrease, red shift $\left(\lambda_{\max }=422 \mathrm{~nm}\right)$ and broadening of the Soret transition (Fig. 1B).

The fluorescence emission spectra of TPPMagA5 and TPPMag, carried out under the same experimental conditions as the UV-vis absorption measurements, strengthen the idea of J-type aggregation, showing decreasing fluorescence emissions and slightly redshifted maximum emission wavelengths $\left(\lambda_{\max }=648 \mathrm{~nm}\right)$ with increasing the water content of $\mathrm{m} / \mathrm{w}$ solutions (Fig. 2). It should be noted that for both compounds the fluorescence excitation spectra overlap the visible absorption spectra, ruling out the formation of fluorescent excited-state species. 


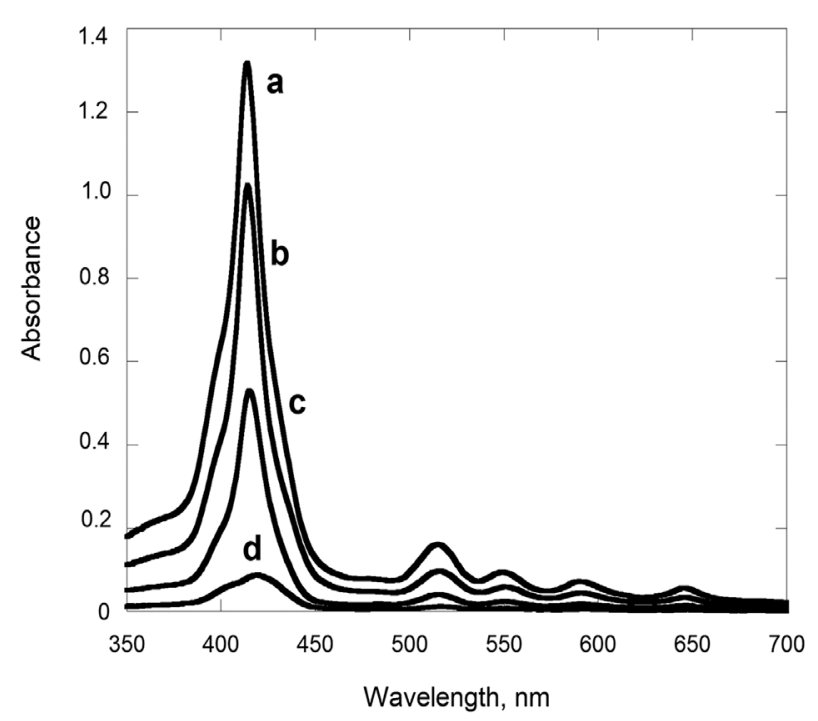

(A)

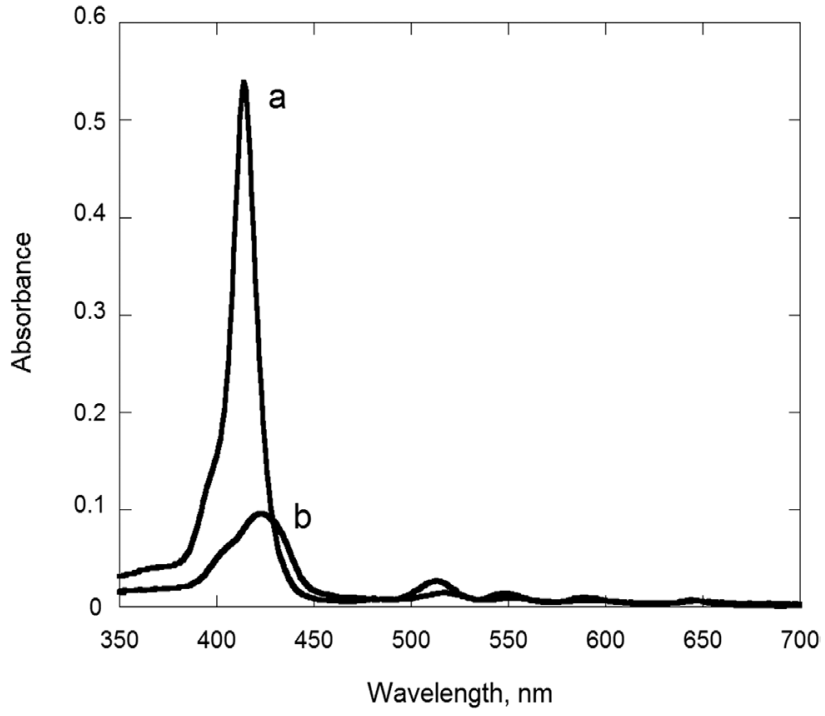

(B)

Fig. 1. Visible absorption spectra. (A) TPPMagA5: (a) $\mathrm{MeOH}$, (b) $\mathrm{MeOH} / \mathrm{H}_{2} \mathrm{O}$ 70:30, (c) $\mathrm{MeOH} / \mathrm{H}_{2} \mathrm{O} 50: 50$, (d) $\mathrm{MeOH} / \mathrm{H}_{2} \mathrm{O} 30: 70$; (B) TPPMag: (a) $\mathrm{MeOH}$, (b) $\mathrm{MeOH} / \mathrm{H}_{2} \mathrm{O} 30: 70$.

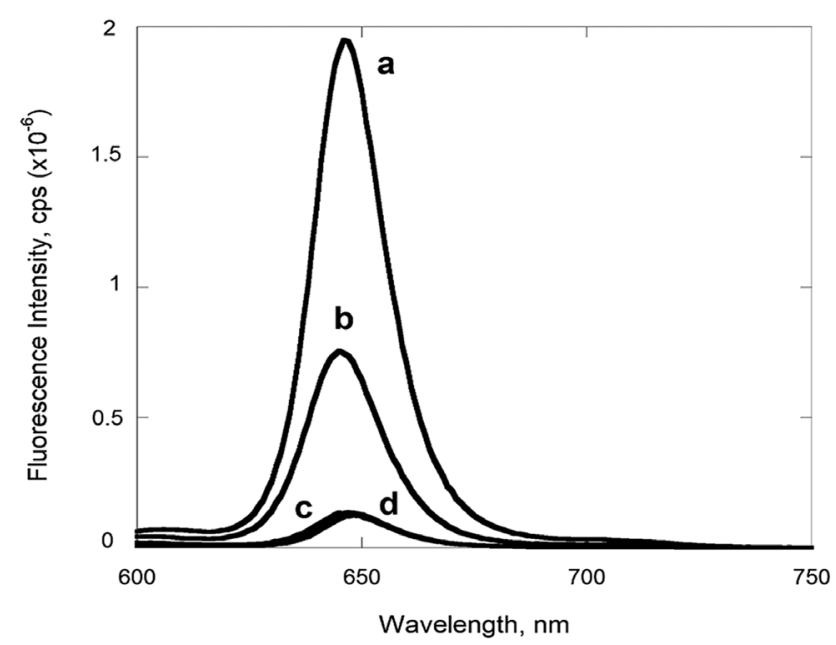

(A)

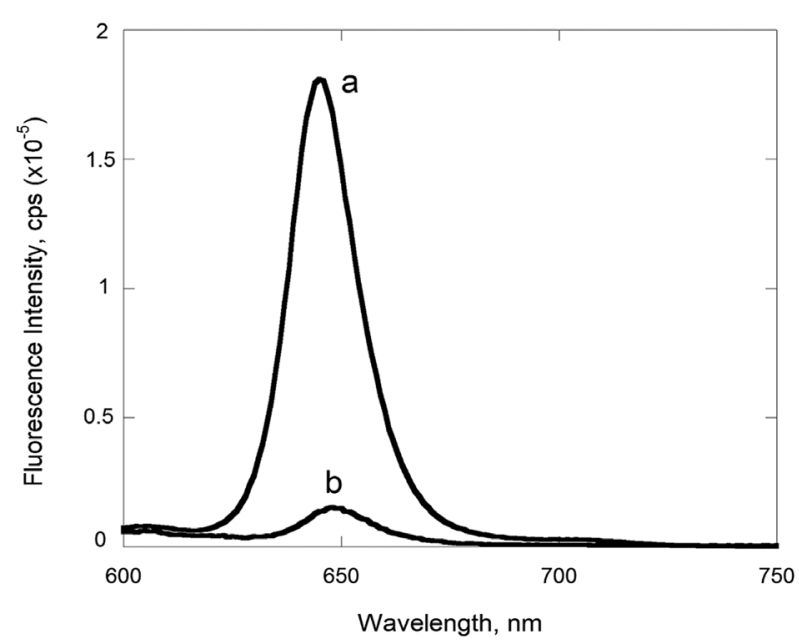

(B)

Fig. 2. Fluorescence emission spectra of (A) TPPMagA5: $\mathrm{MeOH}$ (a), $\mathrm{MeOH} / \mathrm{H}_{2} \mathrm{O}$ 70:30 (b), $\mathrm{MeOH} / \mathrm{H}_{2} \mathrm{O} 50: 50$ (c), $\mathrm{MeOH} / \mathrm{H}_{2} \mathrm{O}$ 30:70 (d); (B) TPPMag: $\mathrm{MeOH}(\mathrm{a}), \mathrm{MeOH} / \mathrm{H}_{2} \mathrm{O} 30: 70$ (b).

The CD spectra of TPPMagA5 and TPPMag in both the amide (200-250 nm) and Soret porphyrin $(350-500 \mathrm{~nm})$ transition regions are reported in Fig. 3. Analyzing the amide region of the spectra, it can be observed that for both compounds the peptide chain attains a $\alpha$-helical conformation under all experimental conditions, i.e. in methanol and in the $\mathrm{m} / \mathrm{w}$ solutions investigated.

Interestingly, under aggregating conditions, i.e. at the higher water fractions of the $\mathrm{m} / \mathrm{w}$ solutions, a bisignate curve, typical of electronic transitions excitonicallycoupled over extended mesoscopic lengths, can be observed in the porphyrin Soret transition region. The positive/negative profile of these bands is characteristic of a helical anti-clockwise arrangement of the peptideporphyrin chiral supramolecular structure. It should be noted that these chiroptical bands showed only negligible intensities in methanol and methanol/water 70/30 solutions.

Detailed CD studies on peptide analogs demonstrated that a direct correlation between the helix screw sense of peptide aggregates and the sign of the induced $\mathrm{CD}$ bands cannot be found, pointing altogether toward a specific role played by the configuration of the first few residues connecting the porphyrin ring to the peptide chain [8].

Intense bisignate $\mathrm{CD}$ bands in the Soret region have often been observed for porphyrin aggregates, and are considered to be diagnostic of the formation of 


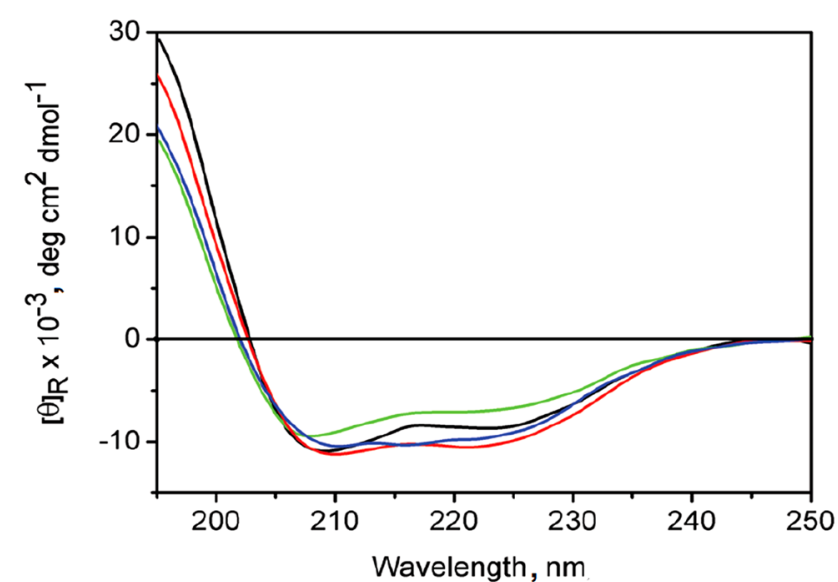

(a)

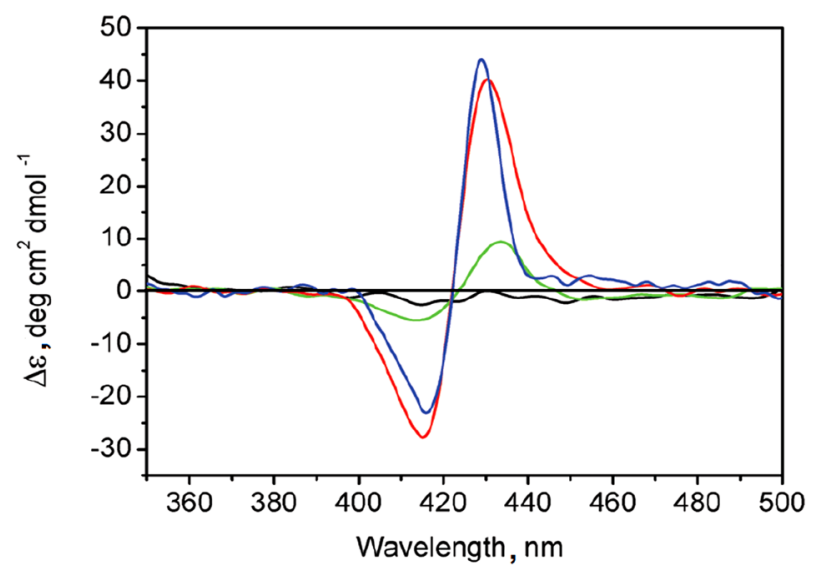

(c)

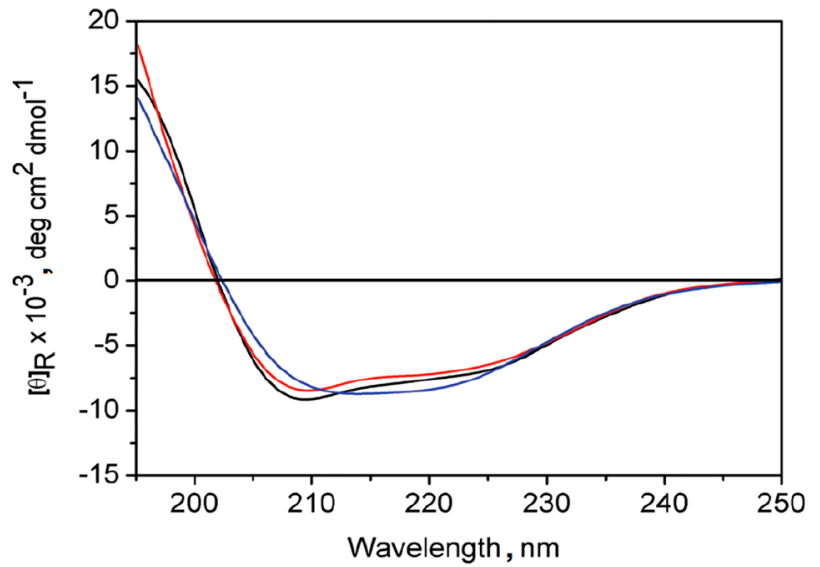

(b)

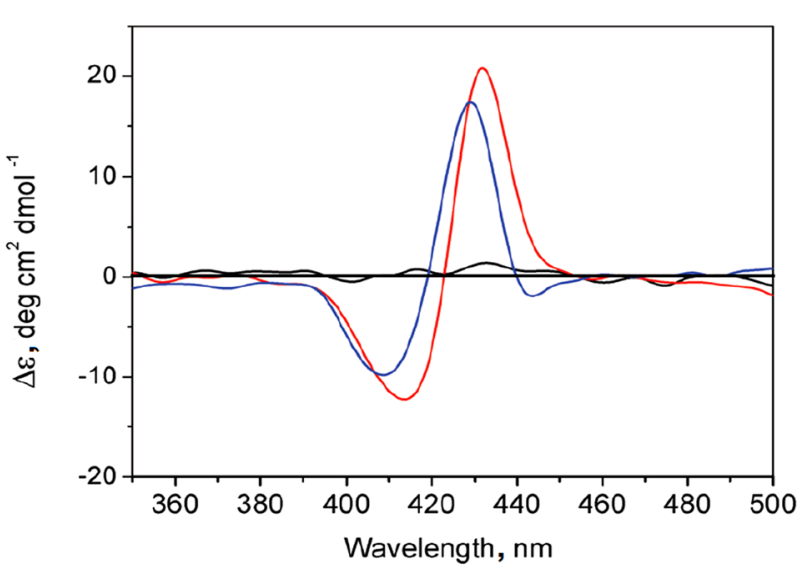

(d)

Fig. 3. CD spectra of TPPMagA5 (a, c) and TPPMag (b, d) in the peptide UV (a, b) and porphyrin Soret transition (c, d) regions. Black: $\mathrm{MeOH}$; green: $\mathrm{MeOH} / \mathrm{H}_{2} \mathrm{O} 70 / 30$; red: $\mathrm{MeOH} / \mathrm{H}_{2} \mathrm{O} 50 / 50$; blue: $\mathrm{MeOH} / \mathrm{H}_{2} \mathrm{O}$ 20/80 (v/v).
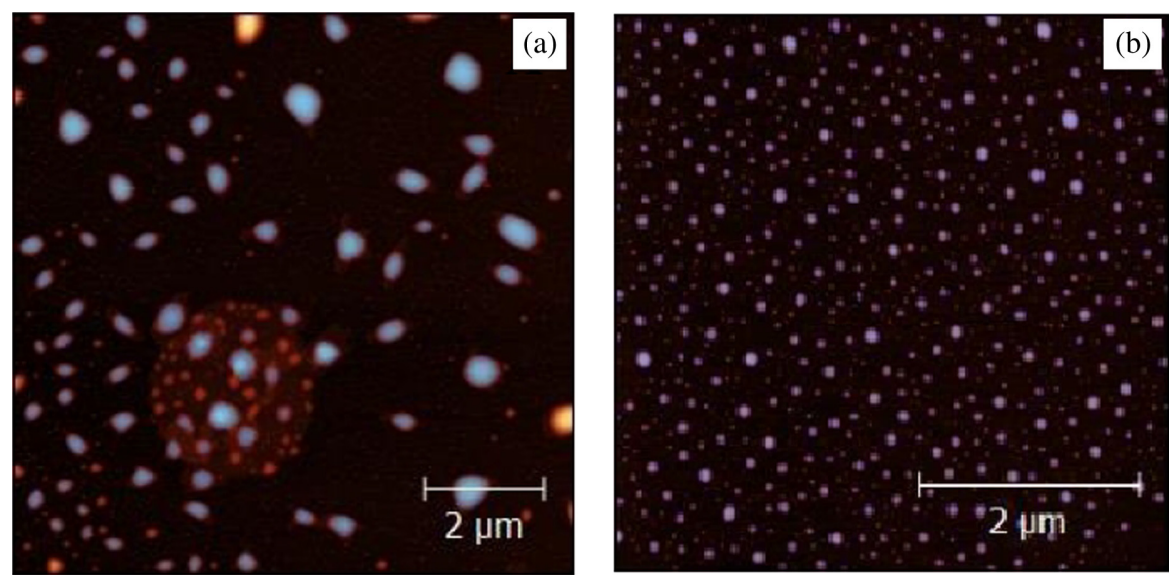

Fig. 4. AFM images obtained from drop casting deposition on mica of micromolar methanol solution of TPPMagA5 (a) and TPPMag (b).

mesoscopic structures $[16,17]$. Interestingly, Castriciano et al. established that bisignate CD signals originated from porphyrin aggregates formed by at least 200 regularly arranged porphyrin macrocycles [18]. It has also been shown that the clockwise/anticlockwise winding of helical coiled/coil porphyrin rods can be controlled by a proper selection of chiral groups functionalizing the porphyrin macrocycle [19]. 

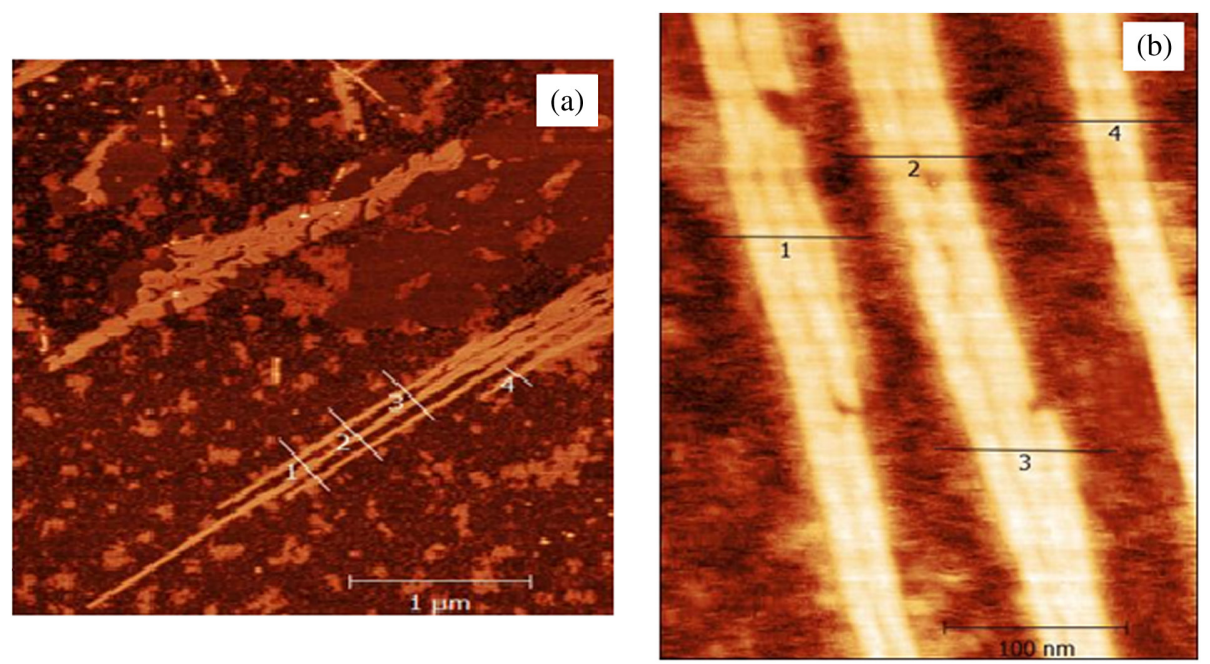

Fig. 5. (a) AFM images obtained from drop casting deposition on mica of micromolar methanol solution of TPPMagA5. (b) Nanometric magnification of the rod structures observed in (a). Thinner filaments could be observed along the 1-4 numbered sections.
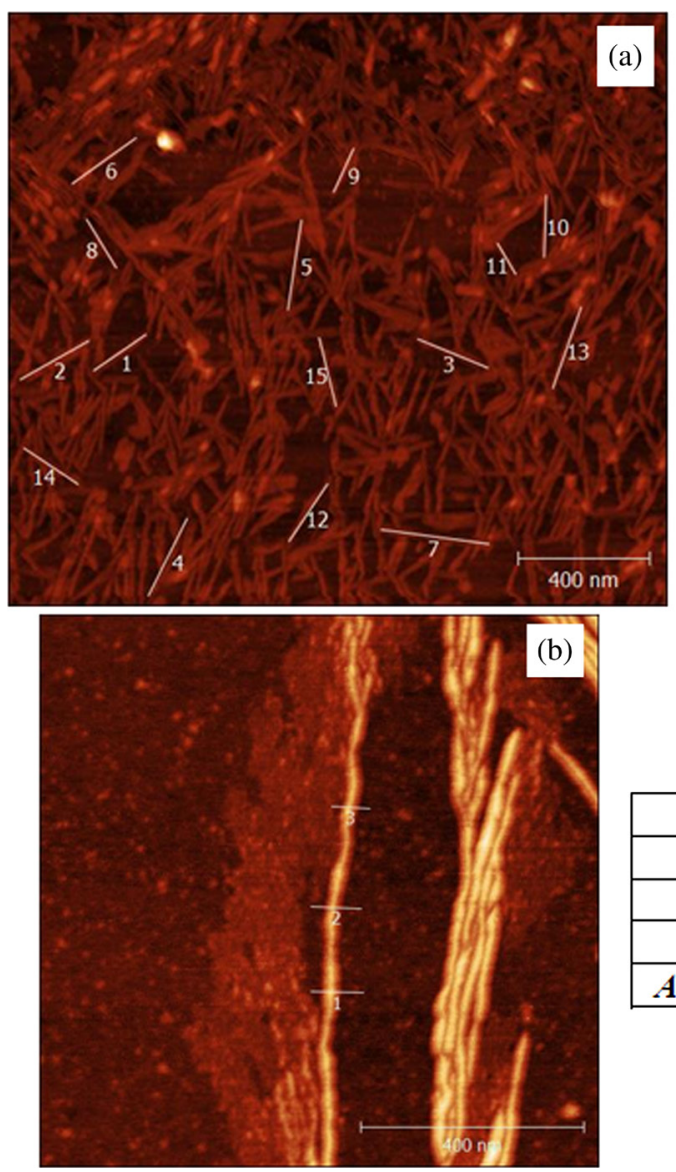

\begin{tabular}{|c|c|}
\hline Fibrils & Length(nm) \\
\hline $\mathbf{1}$ & 192 \\
\hline $\mathbf{2}$ & 238 \\
\hline $\mathbf{3}$ & 230 \\
\hline $\mathbf{4}$ & 262 \\
\hline $\mathbf{5}$ & 276 \\
\hline $\mathbf{6}$ & 238 \\
\hline $\mathbf{7}$ & 330 \\
\hline $\boldsymbol{8}$ & 172 \\
\hline $\boldsymbol{9}$ & 144 \\
\hline $\mathbf{1 0}$ & 188 \\
\hline $\mathbf{1 1}$ & 108 \\
\hline $\mathbf{1 2}$ & 210 \\
\hline $\mathbf{1 3}$ & 261 \\
\hline $\mathbf{1 4}$ & 197 \\
\hline $\mathbf{1 5}$ & 213 \\
\hline Average & $217 \pm 14$ \\
\hline
\end{tabular}

\begin{tabular}{|c|c|c|}
\hline & Width $(\mathbf{n m})$ & Height $(\mathbf{n m})$ \\
\hline $\mathbf{1}$ & 44.6 & 2.2 \\
\hline $\mathbf{2}$ & 43.0 & 2.1 \\
\hline 3 & 43.4 & 2.6 \\
\hline Average & $43.7 \pm 0.7$ & $2.3 \pm 0.2$ \\
\hline
\end{tabular}

Fig. 6. (a): AFM image of TPPMagA5 nanofibers obtained after deposition of TPPMagA5 $0.4 \mu \mathrm{M}$ in methanol/water $30 / 70$ (v/v). (b): Imaging of a TPPMagA5 fiber, showing the intimate structure of intertwined filaments. On the right the morphological features of the nanofibrils imaged in (a) and (b) are reported.

\section{Atomic Force Microscopy experiments}

The morphology of TPPMagA5 and TPPMag aggregates deposited on mica by drop casting from micromolar methanol and 30/70 (v/v) m/w solutions has been characterized by AFM measurements. In the case of deposition from methanol solutions, AFM imaging of both the porphyrin-peptide compounds investigated 


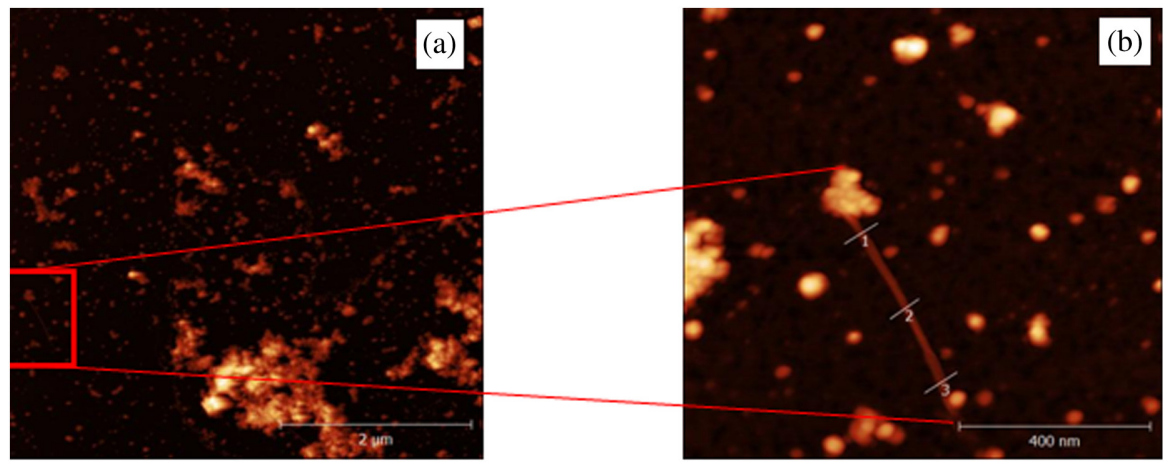

\begin{tabular}{|c|c|c|}
\hline Section & Width $(\mathbf{n m})$ & Height $(\mathbf{n m})$ \\
\hline $\mathbf{1}$ & 26.6 & 3.7 \\
\hline $\mathbf{2}$ & 26.5 & 3.8 \\
\hline $\mathbf{3}$ & 33.1 & 3.6 \\
\hline Average & $28.7 \pm 2.2$ & $3.7 \pm 0.1$ \\
\hline
\end{tabular}

Fig. 7. (a): AFM image of TPPMag agglomerates obtained after deposition on mica of TPPMag in methanol/water 30/70 (v/v). (b): Magnification of the boxed area in Fig. 6a, showing small clusters of globular structures. A single filament connecting two globular structures can also be observed in the Fig. $7 \mathrm{~b}$.

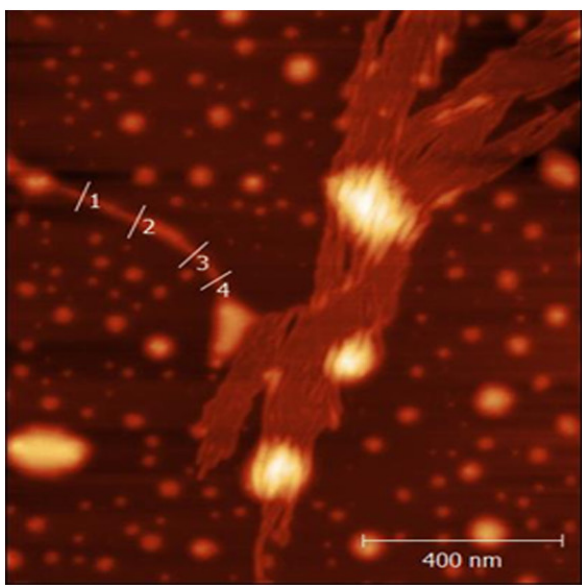

\begin{tabular}{|c|c|c|}
\hline Section & Width(nm) & Height (mm) \\
\hline 1 & 44 & 3.5 \\
\hline 2 & 55 & 3.0 \\
\hline 3 & 52 & 33 \\
\hline 4 & 50 & 2.7 \\
\hline Average & $50 \pm 2$ & $3.1 \pm 0.2$ \\
\hline
\end{tabular}

Fig. 8. AFM image obtained after deposition on mica of TPPMag in methanol/water 30/70 (v/v). A gross fiber structures decorated with bright globules can be clearly envisaged, as well as the thin filament connecting two globular structures. Widths and heights of the latter filament along the numbered sections depicted in the AFM image are also reported.

showed micrometric globular structures of different size distribution. From the data reported in Fig. 4a, for TPPMagA5 an average volume of $4.1( \pm 0.8) \cdot 10^{-3} \mu \mathrm{m}^{3}$ and area of $1.3( \pm 0.2) \cdot 10^{-1} \mu \mathrm{m}^{2}$ can be estimated. Interestingly, TPPMag showed definitely smaller globular structures, the average volume and area being $8.3( \pm 1.2) \cdot 10^{-5} \mu \mathrm{m}^{3}$ and $9.4( \pm 1.1) \cdot 10^{-3} \mu \mathrm{m}^{2}$, respectively (Fig. 4b). Histograms of volumes and areas of the mesoscopic structures formed by the two covalently-linked peptide-porphyrin compounds are reported in the Supporing information (Figs SI1 and SI2). It should be noted that a globular morphology makes it possible to obtain the maximum inner density with the minimum outer surface.

Interestingly, in a restricted area of TPPMagA5 on mica, but not for TPPMag, rods of micrometric length and nanometric width $(70-85 \mathrm{~nm})$ and height $(1.5-1.7 \mathrm{~nm})$ could be observed. At higher resolution these rods appear to be formed by intertwined nanometric filaments (Fig. 5).

Upon drop casting deposition on a mica surface of a methanol/water 30/70 micromolar TPPMagA5 solution, AFM imaging showed the formation of a dense network of fibril structures coating almost homogenously the solid substrate (Fig. 6). From Fig. 6b, it can be seen that the observed fibril structures, having a characteristic length of $217( \pm 14) \mathrm{nm}$, are formed by intertwined thin filaments, showing $43.7( \pm 0.7) \mathrm{nm}$ width and $2.3( \pm 0.2) \mathrm{nm}$ height.

In the case of deposition on mica of a micromolar TPPMag solution carried out under aggregating 
conditions, i.e. in methanol/water 30/70, AFM imaging experiments revealed only the presence of large agglomerates of globular shape (Fig. 7). In Fig. 7b, a thin filament connecting two globular structures can be observed. Analyzing the AFM profiles of the three sections depicted in the figure, the filament was found to have a length of $474 \mathrm{~nm}$, an average width of $29( \pm 2) \mathrm{nm}$, and height of $3.7( \pm 0.1) \mathrm{nm}$.

It should be noted that the generation of fibrils from clusters of aligned globular structures has also been observed in the case of amyloid fibrillization, a process responsible for the onset of degenerative neurological diseases [19]. Interestingly, a similar behavior was observed by us in the case of aggregates of steroid porphyrin derivatives in analogous media [20].

Figure 8 shows a gross TPPMag fibril decorated with globular aggregates and a thin filament connecting two of such globules.

\section{CONCLUSION}

The mesoscopic structures imaged by AFM experiments are the result of the combined effect of two topological motifs: (i) the stacking interaction between the electron-rich porphyrin moieties able to propagate the geometric properties of porphyrin dimers (J- or $\mathrm{H}$-aggregates) over micrometric lengths, and (ii) the helical structure of the peptide chains, functionalizing the porphyrin macrocycle. By hierarchical self-assembly, these structural motifs determine the morphology of the globular or rod-like aggregates propagating the conformational properties of the peptide-porphyrin building blocks to the mesoscopic scale.

It should be noted that the chiral arrangement of the porphyrin supramolecular architectures does not depend on the screw-sense of the peptide helix, but on the conformational properties and hydrophobic nature of the very few residues connecting the porphyrin macrocycle to the peptide chain.

As a consequence, the Alavs. Phesingle-site substitution that distinguishes TPPMagA5 from TPPMag has a deep effect on the morphology of the mesoscopic structures imaged by AFM experiments, although the secondary structure of the two peptide analogues, monitored by CD measurements, definitely appear similar. The Phe-Phe motif has been deeply investigated, due to its capacity to give rise to a rich morphology of peptide nano- and mesostructures, depending on the specific experimental conditions under which the self-assembly process was conducted [21].

The mechanism of fibril formation has been thoroughly investigated for its importance in the early steps of neurodegenerative diseases [22] and in controlled self-assembly of nano- and mesostructures for nanotechnology applications $[23,24]$. In this regard, the imaging of globular structures connected by filament-like segments gives some insight on the potential intermediate structures bridging the globule-to-fibril transition. hese results would be of great importance, among others, for the development of solid-state sensors featuring stereoselective properties, presently pursued in our group [25-28].

\section{Acknowledgments}

This project has received funding from the European Union's Horizon 2020 Research and Innovation programme under the Marie Sklodowska-Curie grant agreement No. 690901.

\section{Supporting information}

Histograms of volumes and areas of the mesoscopic structures formed by the two covalently-linked peptideporphyrin compounds (Figs SI1 and SI2, respectively) are given in the supplementary material. This material is available free of charge via the Internet at http://www. worldscinet.com/jpp/jpp.shtml.

\section{REFERENCES}

1. Ormond AB and Freeman HS. Materials 2013; 6: 817-840.

2. St. Denis TC, Dai T, Izikson L, Astrakas C, Anderson RR, Hamblin MR and Tegos GP. Virulence 2011; 2: 509-520.

3. Dosselli R, Gobbo M, Bolognini E, Campestrini S and Reddi E. ACS Med. Chem. Lett. 2010; 1: 35-38.

4. Chaloin L, Bigey P, Loup C, Marin M, Galeotti N, Piechaczyk M, Heitz F and Meunier B. Bioconjugate Chem. 2001; 12: 691-700.

5. Dosselli R, Tampieri C, Ruiz.Gonzalez R, De Munari S, Ragas X, Sanchez-Garcia D, Agut M, Noneli S, Reddi E and Gobbo M. J. Med. Chem. 2013; 56: 1052-1063.

6. Dosselli R, Ruiz-Gonzales R, Moret F, Agnolon V, Compagnin C, Mognato M, Sella V, Agut M, Noneli S, Gobbo M and Reddi E. J. Med. Chem. 2014; 57: 1403-1415.

7. Moret F, Gobbo M and Reddi E. Photochem. Photobiol. Sci. 2015; 14: 1238-1250.

8. Biscaglia F, Frezza E, Zurlo E and Gobbo M. Org. Biomol. Chem. 2016; 14: 9568-9577.

9. Koti ASR and Periasamy N. Chem. Mater. 2003; 15: 369-371.

10. Eisfeld A and Briggs JS. Chem. Phys. 2006; 324: 376-384.

11. Caruso M, Placidi E, Gatto E, Mazzuca C, Stella L, Bocchinfuso G, Palleschi A, Formaggio F, Toniolo C and Venanzi M. J. Phys. Chem. 2013; 117: 5448-5459.

12. Caruso M, Gatto E, Placidi E, Ballano G, Formaggio F, Toniolo C, Zanuy D, Aleman C and Venanzi M. Soft Matter 2014; 10: 2508-2519. 
13. Caroleo F, Stefanelli M, Magna G, Venanzi M, Paolesse R, Sennato S, Carbone M and Monti D. Org. Biomol. Chem. 2019; 17: 1113-1120.

14. Lettieri R, Monti D, Zelenka K, Trnka T, Drašar P and Venanzi M. New J. Chem. 2012; 36: 1246-1254.

15. Teixeira R, Andrade SM, Vaz Serra V, Paulo PMR, Sánchez-Coronilla A, Neves MGPMS, Cavaleiro JAS and Costa SMB. J. Phys. Chem. B 2012; 116: 2396-2404.

16. Lorecchio C, Venanzi M, Mazzuca C, Lettieri R, Palleschi A, Thi THN, Cardova L, Drasar P and Monti D. Org. Biomol. Chem. 2014; 12: 3956-3963.

17. Simoncini E, Caroleo F, Ceccacci F, Mancini G, Stefanelli M, Paolesse R, Lettieri R, Venanzi M and Monti D. RSC Adv. 2014; 4: 55362-55366.

18. Castriciano MA, Romeo A, De Luca G, Villari V, Monsù Scolaro L and Micali N. J. Am. Chem. Soc. 2011; 133: 765-767.

19. Lettieri R, Cardova L, Gatto E, Mazzuca C, Monti D, Palleschi A, Placidi E, Drasar P and Venanzi M. New J. Chem. 2017; 41: 639-649.

20. Sunde M and Blake CCF. Q. Rev. Biophys. 1998; 31: 1-39.

21. Makam P and Gazit E. Chem. Soc. Rev. 2018; 47: 3406-3420, and references therein.
22. Hamley IW Angew. Chem., Int. Ed. 2007; 46: 8128-8147.

23. Zaytsev DV, Xie F, Mukherjee M, Bludin A, Demeler B, Breece RB, Tierney DL and Ogawa MY. Biomacromolecules 201011: 2602-2609.

24. Pepe-Mooney BJ, Kokona B and Fairman R. Biomacromolecules 2011; 12: 4196-4203.

25. Zelenka K, Trnka T, Tislerova I, Monti D, Cinti S, Naitana ML, Schiaffino L, Venanzi M, Laguzzi G, Luvidi L, Mancini G, Novakova Z, Simak O, Wimmer Z and Drasar P. Chem. - Eur. J. 2011; 17: 1343-1353.

26. Paolesse R, Monti D, Dini F and Di Natale C. (2010) In: Prodi L, Montalti M, Zaccheroni N. (Eds.) Luminescence Applied in Sensor Science. Topics in Current Chemistry, Vol. 300. Springer: Berlin, Heidelberg, 2010, pp. 139-174.

27. Stefanelli M, Magna G, Zurlo F, Caso FM, Di Bartolomeo E, Antonaroli S, Venanzi M, Paolesse R, Di Natale C and Monti D. ACS Appl. Mater. Interfaces 2019; 11: 12077-12087.

28. Paolesse R, Nardis S, Monti D, Stefanelli M and Di Natale C. Chem. Rev. 2017; 117: 2517-2583. 